\title{
The epidemiology of invasive pneumococcal disease in older adults from 2007 to 2014 in Ontario, Canada: a population- based study
}

\author{
Shalini Desai MD MHSc, Michelle E. Policarpio MSc, Kenney Wong MPH, Jonathan Gubbay MBBS, \\ Jill Fediurek BScN MPH, Shelley Deeks MD MHSc
}

Abstract

Background: In Ontario, pneumococcal conjugate vaccines (PCVs) have been sequentially introduced into the publicly funded childhood vaccination program since 2005. A 23-valent polysaccharide pneumococcal vaccine (PPV23) has been routinely recommended for adults aged 65 years and older since 1996. To determine the effect of herd immunity, we examined the epidemiology of invasive pneumococcal disease in adults aged 65 years and older.

Methods: Invasive pneumococcal disease is a provincially reportable disease. We were therefore able to conduct a descriptive epidemiologic analysis that included assessing time trends for patients aged 65 years and older using surveillance data from 2007 to 2014. Using serotype information within the surveillance data, cases were grouped into categories according to vaccine type and periods and then compared using Poisson regression.

Results: A total of 3825 cases of invasive pneumococcal disease were reported among adults aged 65 years and older, for an overall annualized incidence of 25.4 cases per 100000 population. There was a decrease in incidence due to serotypes included in 7-valent PCV (3.0 to 0.7 cases per 100000 population) $(p<0.001)$. For 13-valent PCV serotypes, there was a decrease in incidence between 2011 and 2014 (9.8 to 5.3 cases per 100000 population $(p<0.001)$ ). Serotypes unique to PPV23 and those not included in a vaccine increased from 2.3 to 5.8 and from 2.4 to 7.2 cases per 100000 population, respectively $(p<0.001)$.

Interpretation: In older adults, among serotypes contained in PCVs, we have shown a decrease in incidence of invasive pneumococcal disease. This is likely due to herd immunity from the childhood program. A burden of illness due to unique PPV23 serotypes and those that are not covered by a vaccine exists and has increased over time.

$\sqrt{1}$ he prevention of invasive pneumococcal disease, an important bacterial cause of clinical syndromes such as meningitis, bacteremia and complicated pneumonia, is possible with the use of vaccines. Since the introduction of the 7-valent pneumococcal conjugate vaccine (PCV7), childhood rates of PCV7 serotypes of invasive pneumococcal disease have declined. ${ }^{1-3} \mathrm{~A}$ decrease in nasal carriage of pneumococcal vaccine serotypes among people who have received $\mathrm{CRM}_{197}$-containing conjugate vaccines compared with people who have not received the vaccine has also been described. ${ }^{4-6}$ A number of studies have documented a decrease in adult incidence of PCV7 serotype invasive pneumococcal disease among people who have not received the vaccine owing to herd immunity after childhood vaccination. ${ }^{1,2}$ In a multistate study reported by Whitney and colleagues, reductions in the incidence of invasive pneumococcal disease were seen among adults more than 20 years of age. ${ }^{1}$

The PCV7 vaccine was introduced in January 2005 as part of a publicly funded routine childhood vaccination program for all children born after January $2004^{7}$ in Ontario (population $\sim 13$ million). ${ }^{8}$ The program consisted of a 4 -dose schedule, with doses administered at 2, 4, 6 and 15 months of age. ${ }^{7} \mathrm{~A}$ 10 -valent vaccine (PCV10) replaced PCV7 in October 2009, with a subsequent switch to a 13 -valent product (PCV13) in November $2010 .^{2}$ With this most recent change, Ontario also modified their routine program for infants to receive a total of 3 doses (at 2, 4 and $12 \mathrm{mo}$ ). For infants at high risk, the 4-dose schedule was continued. In adults, PCV13 has not been used in the publicly funded program, with the exception of patients at high risk. ${ }^{9}$ However, since 1996, adults over the age of 65 years have been eligible for a dose of 23-valent polysaccharide pneumococcal vaccine (PPV23), which includes 11 additional pneumococcal serotypes.

Competing interests: Jonathan Gubbay has received a research grant from Pfizer to conduct microbiological surveillance of Streptococcus pneumoniae. No other competing interests were declared.

This article has been peer reviewed.

Correspondence to: Shalini Desai, shalini.desai@phac-aspc.gc.ca CMAJ Open 2016. DOI:10.9778/cmajo.20160035 
We reviewed the epidemiology of invasive pneumococcal disease in Ontario among adults 65 years of age and older over an 8-year period to understand whether there were potential herd effects from the routine infant vaccination program.

\section{Methods}

\section{Case definition}

All cases of invasive pneumococcal disease that meet the following case definition are reportable within the province of Ontario: clinical evidence of invasive disease with laboratory confirmation of infection, which includes isolation of Streptococcus pneumoniae or detection of $S$. pneumoniae DNA by nucleic acid amplification test from a normally sterile site (e.g., blood, cerebrospinal fluid, excluding middle ear) ${ }^{10}$

\section{Surveillance data}

Laboratory confirmation of invasive pneumococcal disease is reported by hospital and private laboratories to public health units. Public health unit staff enter core surveillance data into the integrated Public Health Information System.

Public Health Ontario laboratories send all received isolates of S. pneumoniae from sterile sites to the National Microbiology Laboratory in Winnipeg, Manitoba, for serotyping. In addition, all hospital sites participating in the Toronto Invasive Bacterial Diseases Network send their isolates to the network's laboratory at Mount Sinai hospital in Toronto for serotyping. ${ }^{11}$ Isolates considered not typeable by network methods are forwarded to Winnipeg for further serotyping. Once serotyping is completed, all results are reported centrally to Public Health Ontario. Public Health Ontario assesses results to ensure provincial case definition criteria are met and serotype information is entered into the Public Health Information System on behalf of the respective public health units. ${ }^{10}$

Routine reporting of serotype data to public health began in 2007. Therefore, all cases of invasive pneumococcal disease reported into the integrated Public Health Information System between Jan. 1, 2007, and Dec. 31, 2014, were extracted for this analysis. We included only data from patients 65 years of age and older. Data from patients with noninvasive disease or nonresidents of Ontario were excluded. Age was further divided into 5 - and 10-year age groupings. Data collected included patient sex, episode date, serotype, vaccination information, public health unit and outcome. Specimens with no serotype information were excluded from serotype-specific analysis. Geographic areas were consolidated to 7 health regions that were used for geographic analysis. Vaccination data were collected by the health unit in which a patient resided and were based on records from a doctor or a verbal report by the patient or a delegate.

\section{Laboratory serotyping and serotype groupings}

Serotyping was done using latex pneumococcal antisera (Statens Serum Institute, Denmark) and the capsular swelling Quellung reaction.2

Cases were grouped according to serotypes and vaccine groups (i.e., PCV7, additional PCV13, all PCV13, unique PPV23 and non-vaccine-preventable serotypes). The PCV7 serotype included 4, 6B, 9V, 14, 18C, 19F and 23F. Additional PCV13 serotypes included 1, 3, 5, 6A 7F and 19A. Unique PPV23 serotypes included 2, 8, 9N, 10A, 11A, 12F, $15 \mathrm{~B}, 17 \mathrm{~F}, 20,22 \mathrm{~F}$ and $33 \mathrm{~F}$. All other serotypes not included in a vaccine were considered non-vaccine-preventable.

\section{Statistical analysis}

Incidence rates were calculated using annual population estimates from Statistics Canada (2007-2013) and population projections (2014) obtained through intelliHEALTH Ontario and the Ontario Ministry of Health and Long-Term Care. ${ }^{12,13}$ Descriptive analyses and unadjusted Poisson regression to assess trends over time were done using SAS version 9.3 and Microsoft Excel 2010. In addition, the pre-PCV13 period (January 2007-January 2010) and post-PCV13 period (January 2011-December 2014) were compared using Poisson regression. January 2010 to January 2011 were excluded because that period coincides with introduction of PCV10. An interaction between the vaccine period and years was fitted in the Poisson model with case counts as outcome and population estimates as the offset parameter. The same analysis was repeated to examine time trends for additional PCV13 serotypes. A $p$ value of less than 0.05 was deemed significant.

\section{Ethical approval}

We obtained ethics approval from Public Health Ontario's Ethics Review Board.

\section{Results}

A total of 3825 cases of invasive pneumococcal disease occurred in adults 65 years of age and older during the study period. There was a slight female predominance $(52.0 \%$ of cases were in female patients). Over the 8-year period, the overall annual incidence remained relatively stable, ranging from a high of 28.0 per 100000 population in 2012 to a low of 22.9 per 100000 population in 2014, and there were no significant trends in overall incidence over time $(p=0.4296)$. There was geographic variation in the annualized incidence of invasive pneumococcal disease within the province; the northwest region had the highest annualized incidence (34.7 per 100000 population), whereas the northeast and southwest regions had the lowest (22.3 and 23.7 per 100000 , respectively). Overall, $66.9 \%$ of patients required admission to hospital, and there was a patient fatality ratio of $17.7 \%$.

\section{Age group}

The overall annualized incidence rate of invasive pneumococcal disease was 25.4 per 100000 population among patients 65 years of age and older. When age groups were further subdivided, patients 65-74 years of age had an annualized incidence of 18.7 per 100000 . This increased to 26.5 per 100000 and 50.3 per 100000 for patients 75-84 years of age and more than 85 years of age, respectively. In each of these groups, men had a higher annualized incidence rate than women, and the ratio remained relatively consistent in each age group (Table 1). When viewed by serotype and age, patients in the 
oldest age group (> 85 years of age) had significantly more disease due to all serotypes and by vaccine type than those in the younger age groups. (Table 2).

\section{Serotype data}

Of the 3825 cases identified during the study period, 2778 (72.6\%) had serotyping documented. There was a 20\% annual decrease in the incidence of PCV7-included serotypes between 2007 and 2014, from 3.0 to 0.7 cases per $100000(p<0.001)$ (Figure 1). When analyzing trends over time, the incidence due to PCV13 serotypes significantly increased $(p<0.001)$ from 2007 to 2010 (7.0 to 12.0 cases per 100000 ), followed by a significant decrease $(p<0.001)$ in incidence from 2011 to 2014 (9.8 to 5.3 per 100000$)$. Limiting the analysis to serotypes that were unique to PCV13 (i.e., serotypes $1,3,5,6 \mathrm{~A}, 7 \mathrm{~F}$ and 19A) showed a similar trend, with a significant increase $(p$ $<0.001$ ) in rates between 2007 and 2010 (4.0 to 9.8 cases per $100000)$, with a subsequent significant decrease $(p<0.001)$ in incidence to 4.6 cases per 100000 in 2014. Further serotypespecific analysis showed that within those serotypes included in PCV13, from 2011 to 2014 there was a significant decrease in the incidence per year caused by serotypes $7 \mathrm{~F}(27.9 \%$ decrease), 19A (23.0\% decrease) and 3 (12.7\% decrease).

Additionally, based on an interrupted time series analysis, there was a significant change in the trend before and after the introduction of PCV13 $(p<0.001)$. Changes in trend were significantly different between 2007-2010 and 2011-2013.
For serotypes unique to PPV23, there was a significant increase $(p<0.001)$ in incidence of cases over the study period, with incidence increasing from 2.3 cases per 100000 in 2007 to 5.8 cases per 100000 in 2014. For serotypes not included in any vaccine, there has also been a significant increase $(p<0.001)$ in incidence between 2007 and 2014, from 2.4 cases per 100000 to 7.2 cases per 100000 . Further analysis of unique PPV23 serotypes as well as non-vaccinepreventable serotypes by serotype and 10-year age groupings showed the greatest increase in incidence was in the oldest age group (Figure 2).

\section{Immunization status}

A total of 1223 cases were in patients with a vaccination status documented in the integrated Public Health Information System. Of these patients, $62.4 \%$ were reported as having not received vaccine. Of the $37.6 \%(460 / 1223)$ that had received at least 1 dose of pneumococcal-containing vaccine, $97.0 \%$ (446/460) received PPV23. Interestingly, 26.5\% had illness due to a serotype not covered by PPV23, and $22.6 \%$ had unspecified (i.e., unknown or missing) serotypes. Of the 234 patients who had received PPV23 and who had disease caused by a serotype contained within PPV23, 43.6\% (102/234) had received vaccine more than 5 years before the onset of illness. Among patients who received vaccine, 7.8\% (36/460) had multiple doses of PPV23 and 2.0\% (9/460) received a pneumococcal conjugate vaccine.

\begin{tabular}{|c|c|c|c|c|c|c|}
\hline \multirow[b]{2}{*}{ Age group, yr } & \multicolumn{2}{|r|}{ Women } & \multicolumn{2}{|r|}{ Men } & \multicolumn{2}{|r|}{ Overall } \\
\hline & No. & $\begin{array}{c}\text { Rate } \\
\text { (per } 100000 \text { population) }\end{array}$ & No. & $\begin{array}{c}\text { Rate } \\
\text { (per } 100000 \text { population) }\end{array}$ & No. & $\begin{array}{c}\text { Rate } \\
\text { (per } 100000 \text { population) }\end{array}$ \\
\hline $65-74$ & 715 & 16.7 & 804 & 20.9 & 1519 & 18.7 \\
\hline $75-84$ & 688 & 24.2 & 642 & 29.5 & 1330 & 26.5 \\
\hline$\geq 85$ & 587 & 45.0 & 386 & 60.9 & 973 & 50.2 \\
\hline
\end{tabular}

\begin{tabular}{|c|c|c|c|c|c|c|}
\hline \multirow[b]{2}{*}{ Vaccine type } & \multicolumn{6}{|c|}{ Rate (per 100000 population) by age group } \\
\hline & $65-74 \mathrm{yr}$ & $p$ value & $75-84 \mathrm{yr}$ & $p$ value & $\geq 85 \mathrm{yr}$ & $p$ value \\
\hline All types & 18.7 & Reference & 26.5 & $<0.001$ & 50.3 & $<0.001$ \\
\hline PCV7 & 1.3 & Reference & 1.6 & 0.205 & 4.0 & $<0.001$ \\
\hline Additional PCV13 & 5.3 & Reference & 6.8 & 0.001 & 12.4 & $<0.001$ \\
\hline PCV13 & 6.7 & Reference & 8.5 & 0.001 & 16.5 & $<0.001$ \\
\hline Unique PPV23 & 3.7 & Reference & 4.9 & 0.001 & 9.0 & $<0.001$ \\
\hline NVP & 3.4 & Reference & 5.7 & $<0.001$ & 10.6 & $<0.001$ \\
\hline
\end{tabular}




\section{Interpretation}

Invasive pneumococcal disease causes a substantial burden of disease in adults 65 years of age and older. Annually, an estimated 3000 cases are reported in Canada. The highest incidence is in patients more than 60 years of age (21.9 cases per 100000 population)..$^{14}$ An estimated $90 \%$ of invasive pneumococcal disease in the United States occurs in adults. ${ }^{15}$ When data are further analyzed based on 10-year age groupings, it is evident that the greatest burden of disease is in the oldest adults. ${ }^{16,17}$ Older adults also have a higher case fatality ratio of compared with those in younger age groups ${ }^{17,18}$.

The serotype distribution of invasive pneumococcal disease has changed over the past 8 years. The incidence of non-vaccinepreventable serotypes increased during our study period. In addition, our data show a decrease in disease caused by serotypes contained within the conjugate vaccines. A universal childhood conjugate pneumococcal vaccination program was in place throughout the study period. Therefore, the changes seen in the rates of disease in patients 65 years of age and older are likely due to a herd effect from the childhood program, a decrease in nasal carriage and less exposure to $S$. pneumoniae. This is further substantiated by the serotype-specific changes we saw. As described by other groups, before the introduction of the PCV13 vaccine among children, rates of invasive pneumococcal disease due to serotypes contained within PCV13 increased over time among both children and adults. ${ }^{1,19-22}$ After the introduction of PCV13 to Ontario's childhood vaccination program in November 2010, we saw a decrease in the incidence of serotypes unique to this vaccine among older adults. This is similar to other reports in the literature with sequential PCV7/PCV13 introduction. ${ }^{23-26}$

The PCV13 vaccine was licensed based on an aggregate correlate of protection established for PCV7 serotypes of $0.35 \mu \mathrm{g} /$ $\mathrm{mL}$, as well as an opsonophagocytic antibody (OPA) titre of $1: 8$. Andrews and colleagues ${ }^{27}$ measured serotype-specific cor- relates of protection in infants who had received 2 doses of conjugate vaccine and found that higher cut-offs for protection were need for serotypes $1,3,7 \mathrm{~F}, 19 \mathrm{~A}$ and $19 \mathrm{~F}$, and that an OPA titre of 1:8 was not predictive of protection. Additional evidence from Harboe and colleagues ${ }^{23}$ investigation of Danish population-based laboratory data has shown that since the introduction of pneumococcal conjugate vaccines, serotypes 1 and 3 have not shown a significant change in incidence among patients 65 years of age and older, whereas all other serotypes in PCV13 have. In our study, we saw a significant decrease in the incidence of serotypse $3,7 \mathrm{~F}$ and $19 \mathrm{~A}$ and a decreasing trend for 19F. Our data suggest that based on the incidence of disease, current correlates of protection do provide a basis for estimating protection and that all serotypes contained within PCV13 have shown decreasing trends in incidence among older adults. This difference between what is reported in the literature and what we found could be a true finding, or could be because of less serotype 3 circulating in Ontario.

The PCV13 vaccine is a safe and effective vaccine for older adults. Currently, the National Advisory Committee on Immunization has not recommended that this vaccine be used universally, and no province or territory has implemented universal PCV13 programs among people 65 years of age and older. In contrast, the Advisory Committee on Immunization Practices has made a recommendation to include PCV13 in the routine adult vaccination schedule. ${ }^{28}$ Based on our findings, the herd effect afforded by the routine childhood program may be sufficient for those adults who interact with young children who have received the vaccine. However, this may not be the case for the oldest cohort of adults (patients aged $>85$ years) or adults who have limited interaction with children.

Serotypes unique to PPV23 have increased over our study period. Our observed increase in PPV23 serotype-related disease could be due to a failure to vaccinate (poor vaccine coverage of the universal program). More than $70 \%$ of cases

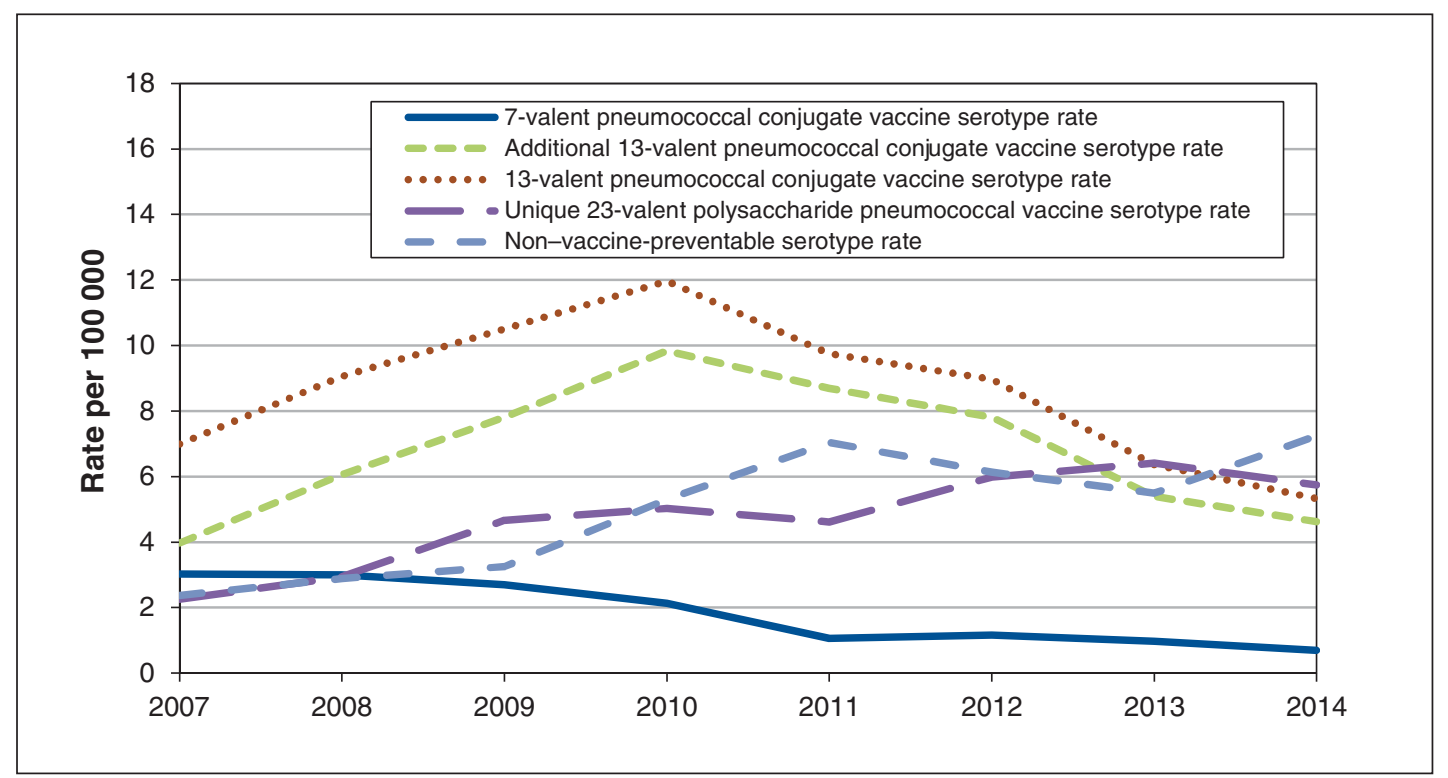

Figure 1: Annual incidence rates of serotype groups by vaccine type among adults 65 years of age and older, in Ontario, Canada, 2007-2014 . 
reported in the integrated Public Health Information System were in patients with no documented receipt of the PPV23 vaccine. However, this could also be due to poor vaccine effectiveness. A comparison of meta-analyses by Melegaro and Edmunds ${ }^{29}$ showed a vaccine effectiveness of $50 \%-80 \%$ against invasive pneumococcal disease in older adults. Effectiveness also varied by age of receipt of vaccine and additional risk factors. Another explanation could be waning immunity - 43\% of patients who had received vaccine had received it more than 5 years before the onset of illness. Morrill and colleagues ${ }^{25}$ describe a national cohort of adults over the age of 50 years in which the percentage of overall cases of pneumococcal infec- tions increased among patients who had received vaccine more than 5 or 10 years earlier. These authors also showed that those who received the vaccine had significantly fewer episodes of invasive pneumococcal disease, despite poor vaccine effectiveness. In Ontario, all adults 65 years of age and older should routinely receive a single dose of PPV23. Vaccine coverage among adults in Ontario is unknown, because there is no comprehensive vaccination registry in the province. National vaccine coverage rates for PPV23 are estimated to be $38.7 \%$ (range $34.5 \%-41.5 \%$ ) among adults aged 65 years and older. ${ }^{30}$ Additional work to promote this vaccine could further decrease the burden of disease due to invasive pneumococcal disease.
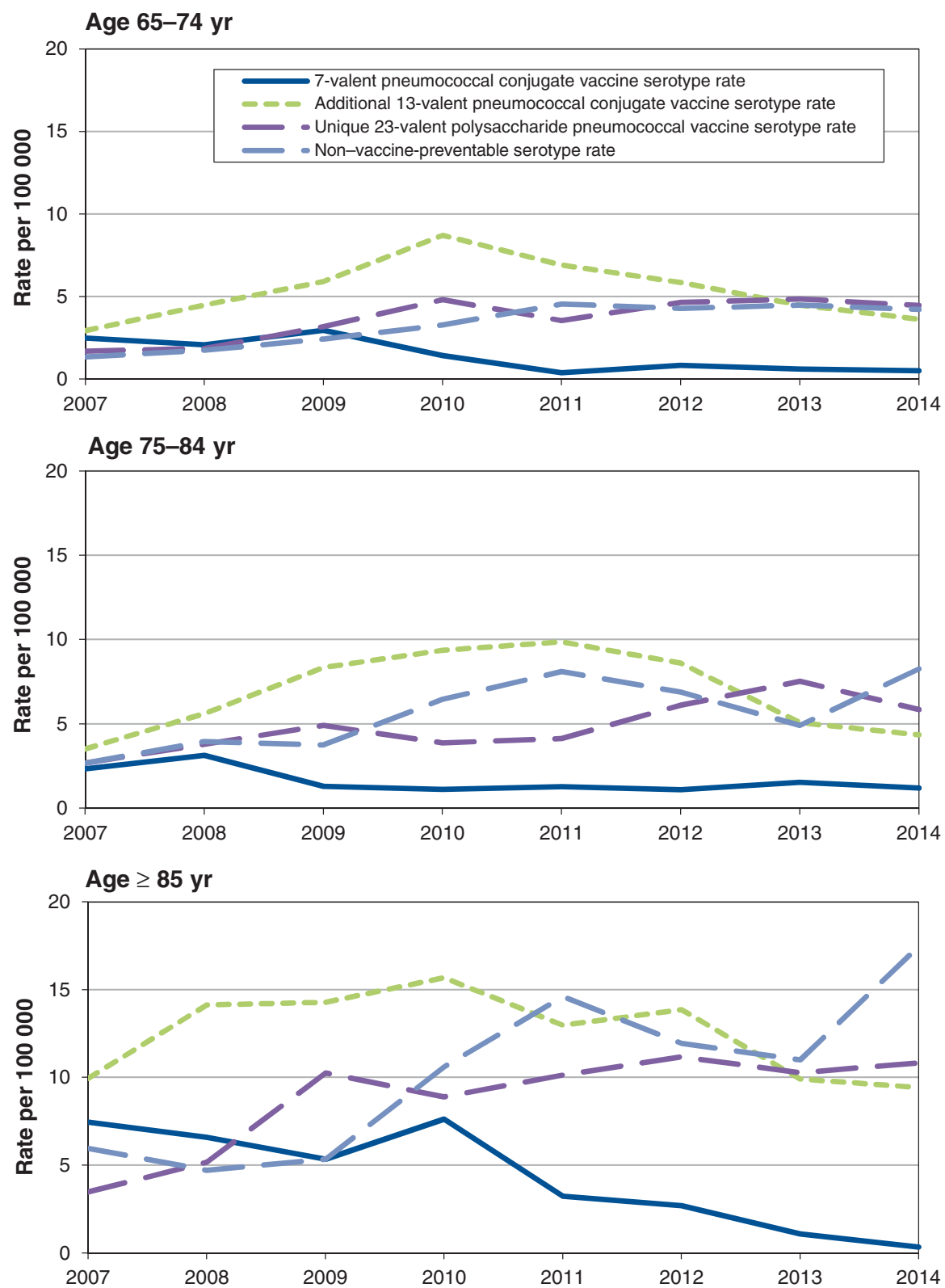

Figure 2: Annual age-specific incidence rates of serotype groups by vaccine type among adults 65 years of age and older (by 10-year groupings) in Ontario, Canada, 2007-2014. 


\section{Limitations}

Although invasive pneumococcal disease is a reportable disease, there is likely under-reporting. Some patients may not have sought medical attention, and some may have received empiric antibiotics without testing. Some variables may also be underreported, particularly those relating to outcome. Twenty-seven percent of our cases were in patients with no serotyping information. This would only impact our findings if these cases were systematically different than those in patients with this information. Our data source did not contain information on risk factors, underlying illness including influenza or ethnicity; these additional variables are known to increase the risk of invasive pneumococcal disease. ${ }^{24}$ Finally, because there is no vaccination registry, assessment of vaccination status was limited by data provided during case investigation.

\section{Conclusion}

Although the overall incidence of invasive pneumococcal disease in older adults has remained relatively stable over time, after the introduction of a childhood pneumococcal conjugate vaccination program, we have seen a decrease in serotypespecific incidence among serotypes contained in conjugate vaccines in older adults. This decrease is likely due to herd immunity. Disease due to serotypes unique to the PPV23 vaccine and those that are not covered by any current vaccine have increased over time. Improving PPV23 vaccine coverage among adults, as well as working toward higher valency conjugate vaccines (i.e., a conjugate vaccine with more than 13 serotypes) or vaccines that target conserved areas of $S$. pneumoniae could lead to further control of invasive pneumococcal disease in this population.

\section{References}

1. Whitney CG, Farley MM, Hadler J, et al. Decline in invasive pneumococcal disease after the introduction of protein-polysaccharide conjugate vaccine. $N$ Engl 7 Med 2003;348:1737-46.

2. Lim GH, Wormsbecker AE, McGeer A, et al. Have changing pneumococcal vaccination programmes impacted disease in Ontario? Vaccine 2013;31:2680-5.

3. Kellner JD, Vanderkooi OG, MacDonald J, et al. Changing epidemiology of invasive pneumococcal disease in Canada, 1998-2007: update from the Calgaryarea Streptococcus pneumoniae research (CASPER) study. Clin Infect Dis 2009; 49:205-12.

4. Obaro SK, Adegbola RA, Banya WA, et al. Carriage of pneumococci after pneumococcal vaccination. Lancet 1996;348:271-2.

5. Mbelle N, Huebner RE, Wasas AD, et al. Immunogenicity and impact on nasopharyngeal carriage of a nonavalent pneumococcal conjugate vaccine. $\mathcal{F}$ Infect Dis 1999;180:1171-6.

6. Gounder PP, Brewster M, Bruce MG, et al. Impact of the pneumococcal conjugate vaccine and antibiotic use on nasopharyngeal colonization by antibiotic nonsusceptible Streptococcus pneumoniae, Alaska, 2000[FIGURE DASH]2010. Pediatr Infect Dis 7 2015;34:1223-9.

7. Update on pediatric invasive pneumococcal disease and recommended use of conjugate pneumococcal vaccines. Can Commun Dis Rep 2010;36(ACS-3):1-30. Available: www.phac-aspc.gc.ca/publicat/ccdr-rmtc/10vol36/acs-3/index-eng. php (accessed 2016 Jan. 25).

8. Population by year, by province, and territory (number) [table]. Ottawa: Statistics Canada; 2015. Available: www.statcan.gc.ca/tables-tableaux/sum-som/101/ cst01/demo02a-eng.htm (accessed 2016 Jan. 25).

9. Statement on the use of conjugate pneumococcal vaccine -13 valent in adults (Pneu-C-13). Can Commun Dis Rep 2013;39(ACS-5):1-52. Available: www. phac-aspc.gc.ca/publicat/ccdr-rmtc/13vol39/acs-dcc-5/index-eng.php (accessed 2016 Jan. 25)

10. Appendix B: provincial case definitions for reportable diseases. Disease - Pneumococcal disease, invasive. Toronto: Ministry of Health and Long-Term Care; 2014. Available: http://www.health.gov.on.ca/en/pro/programs/publichealth/oph_ standards/docs/pneumococcal_cd.pdf (accessed 2016 Jan. 25).
11. Shigayeva A, Rudnick W, Green K, et al. Association of serotype with respiratory presentations of pneumococcal infection, Ontario, Canada, 2003-2011. Vaccine 2016;34:846-53.

12. Population estimates 2005-2013. Ontario Ministry of Health and Long-Term Care, intelliHEALTH Ontario, Statistics Canada; 2014

13. Population projections 2014. Ontario Ministry of Health and Long-Term Care, intelliHEALTH, Ontario Ministry of Finance; 2014.

14. Notifiable diseases online. Ottawa: Public Health Agency of Canada; 2015 Available: http://dsol-smed.phac-aspc.gc.ca/dsol-smed/ndis/charts.php?c=abs (accessed 2016 Jan. 25).

15. Active bacterial core surveillance (ABCs) report - Emerging Infections Program Network, Streptococcus pneumoniae, 2013. Atlanta: Centers for Disease Control and Prevention; 2013.

16. Ruiz LA, Zalacain R, Capelastegui A, et al. Bacteremic pneumococcal pneumonia in elderly and very elderly patients: host- and pathogen-related factors, process of care, and outcome. 7 Gerontol A Biol Sci Med Sci 2014;69:1018-24.

17. Song JY, Choi JY, Lee JS, et al. Clinical and economic burden of invasive pneumococcal disease in adults: a multicenter hospital-based study. $B M C$ Infect Dis 2013;13:202.

18. Lexau CA, Lynfield R, Danila R, et al. Changing epidemiology of invasive pneumococcal disease among older adults in the era of pediatric pneumococcal conjugate vaccine. FAMA 2005;294:2043-51.

19. Grijalva CG, Nuorti JP, Arbogast PG, et al. Decline in pneumonia admissions after routine childhood immunisation with pneumococcal conjugate vaccine in the USA: a time-series analysis. Lancet 2007;369:1179-86.

20. Tsigrelis C, Tleyjeh IM, Huskins WC, et al. Incidence of invasive pneumococcal disease among children after introduction of a 7-valent pneumococcal conjugate vaccine: a population-based study in Olmsted County, Minnesota. Mayo Clin Proc 2009;84:871-5.

21. Meichtry J, Born R, Küffer M, et al. Serotype epidemiology of invasive pneumococcal disease in Swiss adults: a nationwide population-based study. Vaccine 2014;32:5185-91.

22. Navarro Torné A, Dias JG, Quinten C, et al. European enhanced surveillance of invasive pneumococcal disease in 2010: data from 26 European countries in the post-heptavalent conjugate vaccine era. Vaccine 2014;32:3644-50.

23. Harboe ZB, Dalby T, Weinberger DM, et al. Impact of 13-valent pneumococcal conjugate vaccination in invasive pneumococcal disease incidence and mortality. Clin Infect Dis 2014;59:1066-73.

24. Regev-Yochay G, Paran Y, Bishara J, et al. Early impact of PCV7/PCV13 sequential introduction to the national pediatric immunization plan, on adult invasive pneumococcal disease: a nationwide surveillance study. Vaccine 2015;33:1135-42.

25. Morrill HJ, Caffrey AR, Noh E, et al. Epidemiology of pneumococcal disease in a national cohort of older adults. Infect Dis Ther 2014;3:19-33.

26. Bruce MG, Singleton R, Bulkow L, et al. Impact of the 13-valent pneumococcal conjugate vaccine (pcv13) on invasive pneumococcal disease and carriage in Alaska. Vaccine 2015;33:4813-9.

27. Andrews NJ, Waight PA, Burbidge P, et al. Serotype-specific effectivenes and correlates of protection for the 13 -valent pneumococcal conjugate vaccine: a postlicensure indirect cohort study. Lancet Infect Dis 2014;14:839-46.

28. PCV13 (pneumococcal conjugate) vaccine. Atlanta: Centers for Disease Control and Prevention; 2015. Available: http://www.cdc.gov/vaccines/vpd-vac/ pneumo/vac-PCV13-adults.htm (accessed 2016 Jan. 25).

29. Melegaro A, Edmunds WJ. The 23-valent pneumococcal polysaccharide vaccine. Part I. Efficacy of PPV in the elderly: a comparison of meta-analyses. Eur 7 Epidemiol 2004;19:353-63.

30. Vaccine coverage amongst adult Canadians: results from the 2012 adult National Immunization Coverage (aNIC) survey. Ottawa: Public Health Agency of Canada; 2014. Available: http://www.phac-aspc.gc.ca/im/nics-enva/ vcac-cvac-eng.php (accessed 2016 Jan. 25).

Affiliations: Immunization and Vaccine Preventable Diseases Division (Desai, Policarpio, Wong, Fediurek, Deeks), Public Health Ontario; Public Health Ontario Laboratories (Gubbay), Public Health Ontario; Department of Laboratory Medicine and Pathobiology (Gubbay) and Dalla Lana School of Public Health (Deeks), University of Toronto, Toronto, Ont.

Contributors: Shelley Deeks, Shalini Desai, Jill Fediurek and Jonathan Gubbay conceived and designed the study. Michelle Policarpio and Kenney Wong acquired the data. All of the authors were involved in the analysis and interpretation of the data, and the drafting and revising of the article for important intellectual content. All of the authors approved the final version to be published and agreed to act as guarantors of the work.

Acknowledgements: The authors thank Lennon Li and Jeremy Herring for their assistance with statistical testing and population data, as well as Lauren Ramsay for her assistance with formatting and references.

Supplemental information: For reviewer comments and the original submission of this manuscript, please see www.cmajopen.ca/content/4/3/ E545/suppl/DC1 